\title{
Descrição da (im)polidez no uso dos pronomes pessoais nas gramáticas da língua japonesa escritas por estrangeiros no século XIX: uma abordagem historiográfica
}

\author{
Olivia Yumi Nakaema \\ Universidade de São Paulo (USP), São Paulo, São Paulo, Brasil \\ olivia.nakaema@usp.br \\ https://orcid.org/0000-0002-2514-6167
}

\section{DOI: $\mathrm{http://dx.doi.org/10.21165/el.v47i1.2024}$}

\section{Resumo}

Com base na metodologia de análise da Historiografia Linguística proposta por Koerner (1989) e Swiggers (2005[2004]), o objetivo deste trabalho é analisar o tratamento dos pronomes pessoais de $1^{\mathrm{a}}$ pessoa nas principais gramáticas da língua japonesa escritas por estrangeiros no século XIX e investigar como o tema da (im)polidez foi abordado. Juntamente com a análise do contexto social, apresentam-se as mudanças linguísticas correspondentes aos pronomes pessoais e observam-se movimentos de continuidade e descontinuidade em relação às "técnicas de análise e aos métodos de apresentação de dados" (SWIGGERS, 2005[2004]) utilizados nas fontes primárias em estudo.

Palavras-chave: (im)polidez; língua japonesa; pronomes pessoais.

\section{Description of (un)politeness in the use of personal pronouns in Japanese grammars written by foreigners in the 19th century: a historiographical approach}

\begin{abstract}
Based on the methodology of analysis of the Linguistic Historiography proposed by Koerner (1989) and Swiggers (2005[2004]), the objective of this work is to analyze the treatment of the personal pronouns of $1^{\text {st }}$ person in the main Japanese Language grammars written by foreigners in the nineteenth century, and also investigate how the (un)politeness was approached. Along with the analysis of the social context, we present the linguistic changes corresponding to the personal pronouns and continuity and discontinuity movements observed in relation to "techniques of analysis and data presentation methods" (SWIGGERS, 2005[2004]) used in the primary sources under study.
\end{abstract}

Keywords: (un)politeness; Japanese language; personal pronouns. 


\section{Introdução}

A polidez e a impolidez (doravante "(im)polidez") na língua japonesa têm chamado muito a atenção dos estudiosos da língua ao longo da história dos estudos gramaticais japoneses. No século XVII, por exemplo, o padre português João Rodrigues (1561-1634) elaborou a primeira descrição das formas de (im)polidez nas obras Arte da lingoa de Iapam (Nagasaki, 1604-1608) e Arte breve da lingoa Iapoa (Macau, 1620).

No século XIX, esse tema continuou a chamar a atenção dos estudiosos gramaticais estrangeiros ao redescobrirem as gramáticas missionárias de Rodrigues na Europa por meio da tradução para a língua francesa por M. Charles Landresse (biodatas desconhecidas), obra intitulada Éléments de la Grammaire Japonaise, Par le P. Rodriguez (Paris, 1825). Nesse momento, o tema da (im)polidez esteve presente nas principais gramáticas escritas por estrangeiros na Europa e no Japão.

A (im)polidez da língua japonesa está localizada em diversas classes de palavras, principalmente nos pronomes, verbos e palavras flexionáveis (jodôshi). Devido à enorme quantidade de palavras que expressam (im)polidez, neste trabalho, não se pode abordar todas $^{2}$. Desse modo, ainda que outros pronomes também expressem (im)polidez, restringe-se apenas aos pronomes pessoais de $1^{\mathrm{a}}$ pessoa nas seguintes fontes primárias do século XIX, que se destacam pelo critério de importância e recepção ${ }^{3}$ para os estudos gramaticais posteriores:
1) Éléments de la grammaire japonaise par le P. Rodriguez, de Landresse (1825) ${ }^{4}$;
2) Colloquial Japanese (1863), de Samuel Robbins Brown';

\footnotetext{
${ }^{1}$ Aqui adota-se o termo "(im)polidez" para abarcar tanto o fenômeno da polidez quanto da impolidez. Define-se aqui polidez, no sentido amplo, como o fenômeno linguístico comumente chamado de "expressões de tratamento" (em japonês, taigū hyōgen), cuja definição de tratamento é a "distinção no uso da língua tendo como base as relações sociais" (KIKUCHI, 2011, p. 197). Ou seja, aqui polidez corresponde ao uso positivo da língua de acordo com as relações sociais entre os sujeitos. E impolidez corresponde ao uso negativo da língua. Segundo Kikuchi (2011, p. 202-215), as "expressões de tratamento" podem ser classificadas de acordo com o referente, podendo ser de uso positivo ou negativo. Se o referente for o interlocutor, há dois tipos: expressão de polidez em sentido estrito (teineigo) e expressão de polidez ou formalidade (teichôgo). Se o referente for o conteúdo do enunciado, há dois tipos: expressão de respeito (sonkeigo) e expressão de modéstia (kenjôgo). E se o referente for o interlocutor ou conteúdo de enunciado, há dois tipos: expressão de intimidade/carinho (shin'aigo) e expressão depreciativa (higo). Desse modo, neste trabalho, o termo "(im)polidez" abrange todos esses tipos de "expressões de tratamento".

${ }^{2}$ De acordo com Kikuchi (2011, p. 202), na língua japonesa o tratamento é "expresso em praticamente todas as classes de palavras, sendo a principal a dos verbos".

${ }^{3}$ Neste trabalho, o critério de importância e recepção está relacionado ao fato de uma obra ter tido um amplo alcance sobre pessoas engajadas nos estudos sobre a língua, influenciando estudiosos e sendo influente sobre obras gramaticais posteriores no Japão e em outros lugares.

${ }^{4}$ M. Charles Landresse (biodatas desconhecidas), sinólogo e membro da Société Asiatique, traduziu a gramática do padre português João Rodrigues (Arte da lingoa de Iapam, Nagasaki, 1604), publicando a obra Éléments de la Grammaire Japonaise em 1825. Apesar de ser considerada pouco consistente por críticos japanólogos posteriores, como o britânico Satow e o francês Maës Hubert (1938-1977), a obra pode ser considerada a responsável pela redescoberta da língua japonesa pela Europa no século XIX, uma vez que era mais acessível, tanto pelo idioma quanto pela quantidade de exemplares disponíveis, do que a própria obra de Rodrigues.

${ }^{5}$ O missionário britânico imigrado aos EUA Rev. Samuel Brown (1810-1880) chegou ao Japão em 1859 e atuou como professor de inglês. Sua gramática do japonês coloquial é a primeira gramática escrita por um ocidental em terras japonesas no século XIX. Essa obra é de grande importância, pois, além de ser a primeira gramática do japonês escrita em inglês, chegou às mãos de diplomatas ingleses, como Ernest Satow, que escreveu sua própria gramática coloquial em 1873.
} 
3) A Japanese Grammar (translated from Dutch, 1868), de Johann Joseph Hoffmann ${ }^{6}$;

4) Kuaiwa Hen, twenty-five exercises in Yedo colloquial for the use of students (1873) e An English-Japanese Dictionary of Spoken Language (1875), de Ernest Mason Satow $^{7}$;

5) A Short Grammar of the Japanese Spoken Language ( $1^{\mathrm{a}}$ ed. $1869 ; 4^{\mathrm{a}}$. ed. 1888) e A Grammar of the Japanese Written Language ( $1^{\mathrm{a}}$ ed. $1872 ; 3^{\mathrm{a}}$ ed. 1904), de William George Aston ${ }^{8}$;

6) A Handbook of Colloquial Language ( $1^{\mathrm{a}}$ ed. 1888; $2^{\mathrm{a}}$ ed. 1889), de Basil Hall Chamberlain ${ }^{9}$.

Tendo em vista que os estudos historiográficos acerca da (im)polidez na língua japonesa permitem observar um fenômeno linguístico de grande relevância nas descrições escritas por estrangeiros, que desde Rodrigues (1604) destacam uma enorme quantidade de expressões de (im)polidez nessa língua, propõe-se aqui, neste trabalho, analisar o tratamento dos pronomes de $1^{\mathrm{a}}$ pessoa nas fontes primárias mencionadas anteriormente. Essa delimitação se justifica uma vez que muitas línguas ocidentais não possuem marcas de (im)polidez no emprego do pronome de $1^{\mathrm{a}}$ pessoa, o que possibilita uma análise interessante em relação à visão que estrangeiros possuem dos pronomes da língua japonesa. Além disso, como já mencionado, face à enorme quantidade de dados acerca da (im)polidez nas fontes primárias, optou-se por analisar aqui apenas um tipo de pronome. A análise de outras classes gramaticais será realizada em trabalhos posteriores.

\footnotetext{
${ }^{6}$ Johann Joseph Hoffmann (1805-1878) trabalhou em Amsterdã como assistente de Phillipp Franz von Siebold (1796-1866), médico, comerciante e pesquisador de diversas áreas sobre o Japão, e se tornou o primeiro professor de estudos japoneses em uma universidade europeia, na Universidade de Leiden, Holanda. Apesar de nunca ter ido ao Japão, escreveu sua gramática baseando-se em estudos de japonês e chinês realizados na Europa. Assim, devido ao seu pioneirismo nos estudos japoneses, sua obra também merece destaque. Ela foi escrita em holandês e traduzida para o alemão e o inglês, tendo grande repercussão na Europa.

${ }^{7}$ Ernest Mason Satow (1843-1929) fez parte da delegação britânica no Japão ainda no período anterior à Restauração Meiji (1868), tendo sido pioneiro nos estudos linguísticos da língua japonesa. Teve contato com o missionário Brown, de quem recebeu a gramática coloquial publicada em 1873, e escreveu obras que registraram a língua japonesa falada no momento de transição entre os períodos Edo e Meiji. Posteriormente, influenciou os autores de gramáticas da língua japonesa, como Aston e Chamberlain, com quem trocava correspondências constantemente.

${ }^{8}$ William George Aston (1841-1911), assim como Satow, também foi nomeado intérprete da delegação britânica no Japão e tornou-se cônsul e secretário da Delegação Britânica em Tóquio. Também realizou traduções de obras clássicas da literatura japonesa para o inglês e publicou duas gramáticas em 1868 e 1872 , com base nas obras de Satow. Essas obras são muito semelhantes à de Chamberlain, que as usou como base para sua própria gramática.

${ }^{9}$ Basil Hall Chamberlain (1850-1935) chegou ao Japão em maio de 1873 e tornou-se rapidamente um conhecedor da língua. Destacou-se como estudioso e tornou-se professor na Academia Naval Japonesa em 1874 e professor de Língua Japonesa e Filologia na Universidade Imperial de Tóquio de 1886 a 1911, na qual também se tornou professor emérito. Foi professor de importantes linguistas japoneses, como Ueda Kazutoshi (1867-1937), entre outros, e foi pesquisador não só da língua japonesa, mas também dos dialetos do povo Ainu (originários da atual região de Hokkaidô) e de Ryûkyû (atual arquipélago de Okinawa). A pedido do Ministério da Educação do Japão, escreveu a gramática da língua japonesa A Handbook of Colloquial Japanese ( $1^{\mathrm{a}}$ ed., 1888), que influenciou diretamente seus alunos, passando a ser considerado o "pai da linguística japonesa". Desse modo, sua gramática é extremamente relevante por recepcionar os trabalhos de Satow e Aston, além de influenciar gramáticos estrangeiros e japoneses posteriores.
} 


\section{A sociedade japonesa e a (im)polidez: mudanças sociais e linguísticas expressas por meio de pronomes pessoais}

A história dos estudos linguísticos pode ser representada pelo modelo Continuidade vs. Descontinuidade proposto por Koerner (1989) ${ }^{10}$, que possibilita entender o porquê de uma linha de investigação passar a se destacar, enquanto outra, tida como tradicional, passa a ser ignorada ${ }^{11}$ :

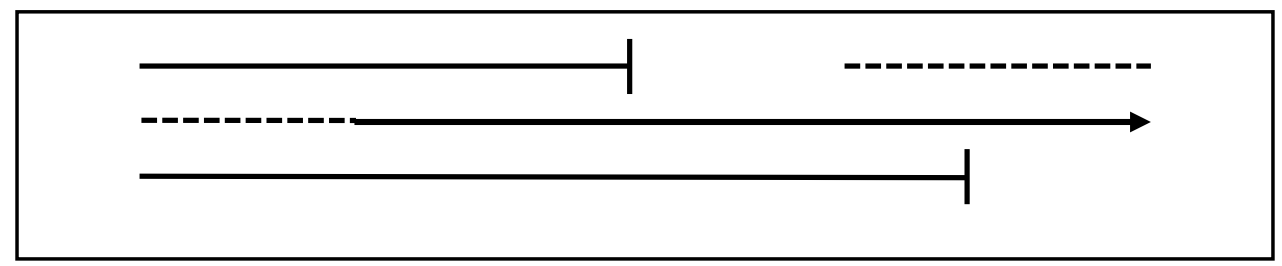

Figura 1. Modelo de Descontinuidade vs. Continuidade

Fonte: Koerner (1989, p. 53)

Esse modelo parece ser adequado para os estudos historiográficos que descrevem os fenômenos linguísticos como a polidez, uma vez que indica a possibilidade da descontinuidade de determinadas tradições. Desse modo, os estudos acerca da (im)polidez expressa nos pronomes pessoais da língua japonesa no século XIX também podem valer-se desse modelo, tendo em vista a presença dos movimentos de continuidades e descontinuidades das formas de descrição utilizadas em cada gramática. Por exemplo, com relação ao uso da língua portuguesa para descrever a língua japonesa, pode-se observar que havia uma tradição durante os séculos XVI e XVII, mas esta acabou sendo deixada de lado em decorrência da decadência das políticas ultra marítimas portuguesas. Posteriormente, no século XX, com as relações imigratórias entre Brasil e Japão, e o aumento dos laços de amizade entre japoneses e brasileiros, novamente a língua portuguesa volta a ser a língua descritora nas gramáticas do japonês. Desse modo, esse modelo parece se adequar bem aos estudos de investigação linguística acerca da polidez do japonês, uma vez que as tradições se apresentam de forma contínua e descontínua ao longo do tempo.

Assim, por meio desse modelo de descontinuidades, pode-se analisar a história dos estudos linguísticos acerca dos pronomes pessoais da língua japonesa. Por isso, para entender a organização dos pronomes pessoais no século XIX, faz-se necessário abordar o contexto histórico e a mudança linguística relativa a esse tema.

A história dos estudos linguísticos do Japonês é comumente dividida da seguinte forma (FRELLESVIG, 2011[2010]): (1) Japonês Antigo (anos 700 a 800): corresponde aproximadamente ao período político Nara (712-794); (2) Japonês Medieval Antigo (anos 800 a 1200): corresponde aproximadamente ao período político Heian (794-1185);

\footnotetext{
${ }^{10}$ Koerner (1989, p. 51) apresenta vários modelos para entender a Historiografia da Linguística. No modelo tradicional, o conhecimento científico é cumulativo e não começa sempre do ponto inicial em determinado tempo. Outro modelo proposto é o que o autor chama de "Mainstream vs. Undercurrent Model" (Modelo de grande aceitação vs. marginal), onde há alternância entre uma linha de investigação receber oficial aceitação pela maioria de uma comunidade e encontrar-se em situação marginalizada, isto é, uma linha de investigação é aceita como o centro das atenções sobre outra linha em determinado período de tempo.

${ }^{11}$ Como exemplo desse modelo, Koerner (1989, p. 53-54) cita a tradição de Linguistique Générale de Saussure, que veio de Bréal a Saussure e continuou até Hjelmslev (1928), tendo sido retomada por Chomsky em Cartesian Linguistics (1966).
} 
(3) Japonês Medieval Recente (anos 1200 a 1600): corresponde aproximadamente ao período político Kamakura (1185-1333) e Muromachi (1333-1573); (4) Japonês Moderno (1600 em diante): corresponde aproximadamente aos períodos políticos Edo (1603-1868), Meiji (1868-1912), Taishô (1912-1926), Shôwa (1926-1989) e Heisei (a partir de 1989).

Para melhor apresentar com precisão a mudança linguística no século XIX, divide-se o período denominado Japonês Moderno em quatro subperíodos: (4.1) Japonês Moderno do Período Edo; (4.2) Japonês Moderno do Período Meiji; (4.3) Japonês Moderno do século XX até 1945; (4.4) Japonês Moderno do século XX após 1945.

Neste trabalho, a fim de analisar os pronomes pessoais na língua falada no século XIX, interessa observar o contexto dos dois primeiros períodos do Japonês Moderno, isto é, Períodos Edo e Meiji, que correspondem à época de publicação das gramáticas analisadas. O Período Edo (primeiro período referente ao japonês moderno) é marcado pela pacificação militar proporcionada pelo clã Tokugawa e pela sociedade basicamente dividida em quatro estratos principais: samurais, agricultores, artesãos (incluem-se aqui os profissionais liberais) e comerciantes (DOI, 1979[1957]). Além dessas classes, havia também o imperador e a nobreza. A ascensão social era muito difícil, salvo raríssimas exceções, e havia uma grande distância entre os samurais e as demais classes, o que se refletia também na forma falada: os samurais utilizavam mais palavras de origem chinesa e expressões de polidez (honoríficos e modéstia ${ }^{12}$ ) (DOI, 1979 [1957]). Nesse período, os samurais não estavam exclusivamente dedicados às práticas militares, uma vez que o período de guerras intensas havia cessado, mas realizavam atividades administrativas e precisavam ir à capital Edo para servir ao clã Tokugawa, representado pela figura máxima do xogum (DOI, 1979[1957]). Assim, a partir do contato entre diversos dialetos de distintas regiões do Japão e da forma falada na capital, passa a surgir a língua de Edo, resultado dessa mistura.

Assim, no Período Edo, os pronomes pessoais também refletiam essa estratificação social ${ }^{13}$. Por exemplo, o pronome de $1^{\mathrm{a}}$ pessoa ORE era usado por homens e por mulheres de alta classe social (SATÔ, 1992[1973]). A partir da segunda metade do Período Edo, ore passou a ser usado principalmente por homens e aparecia também nas

\footnotetext{
${ }^{12}$ Honoríficos ou respeito corresponde ao sonkeigo, que expressa respeito à pessoa que é mencionada no conteúdo do enunciado, por meio da "elevação" do interlocutor. Modéstia ou humilhação corresponde ao kenjôgo, que também expressa respeito à pessoa que é mencionada no conteúdo do enunciado, mas por meio do "rebaixamento" do enunciador.

${ }^{13}$ Pronomes pessoais de $1^{\text {a }}$ pessoa utilizados no Período Edo: - ORE（おれ），KOCHI（こち）, KOCHITO/KOCHITORA（こちとにちとら）, SESSHA（拙者）, SOREGASHI（それがし）， MIDUKARA（みづから）, MIDOMO（身共）, WATAKUSHI（わたくし）,WATASHI（わた し）,WASHI（わし）, WAGAMI（わが身）,WARAHA（わらは）, URA（うら）, ONCHI（お んち）, WATAI（わたい）, WACCHI（わっち）, WACHIKI（わちき）, entre outros. Pronomes pessoais de $2^{\mathrm{a}}$ pessoa utilizados nesse período: KONATA（こなた）, SONATA（そなた）, ONUSHI/ONOSHI（おぬし/おのし）, NANJI（汝）, SOCHI（そち）, OMAE（お前）, OMEE （おめ元）, OTEMAE（お手前）, KISAMA（貴様），KONASAMA/KONASAN)（こな様になさ ん）, SOSAMA（そ様）, SOCHITO（そちと）, ONORE（おのれ）, UNU（うぬ）, WARE（わ れ）, entre outros. Pronomes de $3^{\mathrm{a}}$ pessoa: ANOSAN（あのさん）, ANATA/ANTA（あなた/あんた）， AITSU（あいつ）, entre outros. (DOI, 1979 [1957]; SATÔ, 1992 [1973]; FRELLESVIG, 2011 [2010]).
} 
variantes ora e oira (SATÔ, 1992[1973]). Os pronomes de $1^{\text {a }}$ pessoa sessha e soregashi eram usados principalmente por samurais (SATÔ, 1992[1973]). O pronome midomo, no Período Edo, era mais usado por samurais (SATÔ, 1992[1973]), mas posteriormente, passou a ser usado também por profissionais liberais das cidades e mulheres $(n k d)$. Os pronomes de $2^{\text {a }}$ pessoa omae, otemae, kisama, konasama(n), sosama e sochito eram formas novas que surgiram no Período Edo (SATÔ, 1992[1973]). omae, originária da variante falada na região de Osaka, era a forma de maior grau de polidez no Período Edo, superando a forma konata (SATÔ, 1992[1973]). Desse modo, cada classe social utilizava uma linguagem distinta, fazendo uso de pronomes pessoais de forma diferente.

O Período Meiji inicia-se com o fim do governo do xogunato Tokugawa, a extinção da classe samurai e o fim das antigas classes sociais. Assim, com a reestruturação social, as formas linguísticas que expressavam (im)polidez também mudam (DOI, 1979 [1957]). Surge então a língua de Tóquio considerada padrão, rica em novas expressões que poderiam ser usadas entre falantes de mesmo nível social (SATÔ, 1992[1973]). Com relação aos pronomes pessoais, essas mudanças sociais também podem ser observadas. Por exemplo, os pronomes de $1^{\text {a }}$ pessoa SOREGASHI e MIDOMO, entre outros, antes utilizados por samurais, deixam de ser usados e foram substituídos pelo pronome BOKU de uso geral (SATÔ, 1992[1973]). O pronome OMAE deixa de ser utilizado para se referir a pessoas de nível hierárquico superior (SATÔ, 1992[1973]). Também surge o pronome de $3^{\mathrm{a}}$ pessoa feminino KANOJO, correspondente ao "she" da língua inglesa (SATÔ, 1992[1973]). Ante a essas mudanças, pode-se notar que o desaparecimento da classe dos samurais fez com que o uso do pronome pessoal se tornasse mais igualitário e sem tantas expressões que distinguissem as pessoas entre si.

Desse modo, após a breve exposição das mudanças linguísticas e do contexto social, passa-se à análise dos pronomes de $1^{\mathrm{a}}$ pessoa nas fontes primárias já mencionadas.

\section{Análise da capa técnica referente aos pronomes pessoais}

Como método de análise e comparação das gramáticas escritas sobre a língua japonesa, faz-se uso da metodologia de análise por "capas" (ou domínios ou camadas ${ }^{14}$ ) propostas por Swiggers (2005[2004], p. 133-134, tradução nossa) ${ }^{15}$. Esse autor define quatro capas, como apresentamos a seguir: (1) Capa teórica: "corresponde à visão global da linguagem, à concepção das tarefas e do status da linguística"; (2) Capa técnica: "inclui as técnicas de análise (linguístico/ gramatical) e os métodos de apresentação de dados"; (3) Capa documental: "corresponde à documentação linguística e filológica (número de línguas, tipos de fontes e de dados) sobre o qual se baseia o estudo

\footnotetext{
${ }^{14}$ Swiggers utilizou o termo "capas" em texto em espanhol, no sentido de "camadas" do conhecimento. Alguns autores, como Coelho e Hackerott (2012), já utilizaram o termo "domínios", mas atualmente o termo mais utilizado pelo Centro de Documentação em Historiografia Linguística da Universidade de São Paulo é "camadas".

${ }^{15}$ Essa proposta de Swiggers foi apresentada no IV Congresso Internacional de la SEHL, em 2004, e publicada em Actas de 2005. Coelho e Hackerott (2012) explicam que, como analisar historiograficamente o pensamento linguístico é muito complexo, a proposta de Swiggers de entender a sucessão de modelos teóricos divididos em "capas" possibilita compreender onde se encontram as transformações e onde se encontram os pontos estáveis. Assim, uma mudança pode ser verificada apenas em um dos domínios ou em mais de um deles. Nesse último caso, pode-se até mesmo verificar uma "revolução", no sentido proposto por Kuhn.
} 
linguístico"; (4) Capa contextual/ institucional: "corresponde ao contexto cultural e institucional da reflexão e da prática linguística".

Neste trabalho, analisa-se a capa técnica acerca dos pronomes pessoais de $1^{\mathrm{a}}$ pessoa, uma vez que essa metodologia se mostra produtiva para investigar as técnicas gramaticais de análise e métodos de apresentação de dados, isto é, da terminologia empregada (casos, declinações, etc.) e da comparação com outras línguas.

\subsection{Uso de idiomas estrangeiros}

Nas gramáticas em análise, nota-se que a língua francesa só é utilizada como língua descritora em Landresse (1825). Já a língua inglesa é a que mais apresenta continuidade, sendo usada em Brown (1863), Hoffmann (1868), Satow (1873 e 1904[1875]), Aston (1888[1869] e 1904[1872]) e Chamberlain (1888). Além dessas línguas, há também o uso do alemão e do holandês por Hoffmann (1868).

Pode-se assim notar o poder político e econômico dos países de língua inglesa no século XIX, em especial da Inglaterra. Antes da abertura dos portos japoneses aos países do Ocidente em 1854, apenas a Holanda podia ter contato com o Japão e só o holandês era visto como importante, sendo utilizado para estudos de medicina, chinês, botânica, etc. No entanto, após essa abertura, outras nações podiam comercializar nos portos japoneses após celebrarem tratados comerciais com o Japão. A presença holandesa diminuiu devido ao enfraquecimento desse país nas relações comerciais e a presença britânica se intensificou, não apenas no Japão, mas em toda a Ásia. Assim, outras línguas passaram também a ser utilizadas nos portos japoneses e, consequentemente, nos estudos linguísticos do Japonês, destacando-se o inglês após a Restauração Meiji (1868) ${ }^{16}$.

\subsection{Uso da classe de palavra "pronome" e a classificação em "pessoa"}

Conforme se observa no Anexo I - Quadro: Tratamento dado aos pronomes pessoais de $1^{a}$ pessoa, nas obras de Landresse (RODRIGUEZ, 1825), Brown (1863), Satow (1873 e 1904[1875]) e Aston (1888[1869] e 1904[1872]), utilizam-se a classe de palavra "pronome" e o termo "pessoas" para distinguir os tipos de pronomes. Nas gramáticas em que não se utiliza os termos "pronomes" e "pessoas", há apresentação das expressões japonesas como equivalentes aos pronomes "I" e "We" da língua inglesa, como em Hoffmann (1868) e Chamberlain (1888).

Por exemplo, em Landresse (RODRIGUEZ, 1825, Livre Premier, p. 9, tradução e grifos nossos), o autor faz uso dos termos "pronom" (pronome) e "pronom de la première personne" (pronome de primeira pessoa) ${ }^{17}$ : “\$48. Como nós havíamos dito, os pronomes primitivos se declinam como os substantivos, ao se ajuntar às mesmas partículas, para os casos e para os nomes. Exemplo: Pronome de primeira pessoa, singular e plural".

\footnotetext{
${ }^{16}$ O intelectual Fukuzawa Yukichi (1835-1901), fundador da Universidade Keio, reflete essa mudança da valorização do idioma holandês para o inglês. Enquanto jovem, ele se dedicou aos estudos de holandês, mas, ao entrar em contato com os estrangeiros no Japão, notou que o inglês era a língua falada entre eles. Ao viajar aos Estados Unidos e Europa, percebeu que o pilar da sociedade moderna ocidental era a língua inglesa (SHUNSAKU, 1993). Então, passou a se dedicar aos estudos desse idioma.

17 “\$48. Ainsi que nous l'avons dit, les pronoms primitifs se déclinent comme les substantifs, en ajoutant les mêmes particules, pour les cas et pour les nombres. Exemple: Pronom de la première personne, singulier et pluriel".
} 
Em Chamberlain (1888, p. 46, tradução e grifos nossos), por exemplo, não se usa o termo "pronome", mas há menção ao pronome "I" (eu $)^{18}$ : "66. O mais usual equivalente para 'I' (eu) é wataküshi, lit. 'selfishness' (eu)".

Desse modo, com base nesses exemplos e também nos apresentados no Anexo I, tem-se que, em todas as gramáticas analisadas, há duas tendências: 1) o uso do termo "pronome", referindo-se diretamente a essa classe gramatical; ou 2) o uso da equivalência aos pronomes de outra língua sem utilizar o termo "pronome", como em Chamberlain. Assim, ainda que não utilize diretamente o nome da classe gramatical, gramáticas como a de Chamberlain indicam a tradução do pronome na língua inglesa, não se afastando da noção de pronome.

\subsection{Uso de "casos" latinos}

Seguindo o modelo da gramática de Rodrigues (1604), Landresse (RODRIGUEZ, 1825 , p. 9, tradução e grifos nossos) faz uso dos casos latinos para descrever os pronomes pessoais de $1^{\mathrm{a}}$ pessoa ${ }^{19}$ :

"Nom. Ware, warewa, je ou moi (eu ou me/mim); warera, nous (nós).

Gen. Wareno ou warega, de moi, ou du mien (meu ou de mim); warerano ou wareraga, de nous, ou des nôtres (nosso ou de nós).

Datif. Wareni, wareye, à moi, pour moi (a mim, para mim); warerani, wareraye, à nous, pour nous (a nós, para nós).

Accus. Warewo, warewoba, moi (me); warerawo, warerawoba, nous (nos).

Ablat. Wareyori, de moi, par moi (de mim, por mim); warerayori, de nous, par nous (de nós, por nós)."

Em Landresse (RODRIGUEZ, 1825), há uso das expressões abreviadas de "nominativo", "genitivo", "dativo", "acusativo" e "ablativo".

Nas demais gramáticas analisadas, há uma descontinuidade no uso dos casos, uma vez que não mais utilizam esse modelo latino, mas sim há uma tendência de aproximação com a gramática de língua inglesa, que não possui casos.

\subsection{Uso de classes sociais}

Ao comparar as gramáticas analisadas, nota-se que há uso de classes sociais em apenas algumas delas, como se apresenta no Anexo I. Interessante notar que esse uso reflete as mudanças por que passavam a sociedade japonesa no século XIX. Por exemplo, com a Restauração Meiji em 1868, extinguiram-se o Xogun, que regia o país em nome do Imperador, e a classe dos samurais. Surgiram no lugar dos samurais funcionários públicos que passaram a residir na capital Tóquio. Desse modo, as gramáticas analisadas descrevem a língua japonesa em cada momento político diferente, o que torna o trabalho

\footnotetext{
18 "66. The most usual equivalent for "I" is wataküshi, lit. " "selfishness".

${ }^{19}$ Nom. Ware, warewa, je ou moi; warera, nous.

Gen. Wareno ou warega, de moi, ou du mien; warerano ou wareraga, de nous, ou des nôtres.

Datif. Wareni, wareye, à moi, pour moi; warerani, wareraye, à nous, pour nous.

Accus. Warewo, warewoba, moi; warerawo, warerawoba, nous.

Ablat. Wareyori, de moi, par moi; warerayori, de nous, par nous.

Gen. Wareno ou warega, de moi, ou du mien (meu ou de mim); warerano ou wareraga, de nous, ou des.
} 
de análise do uso das classes sociais muito interessante para observar essas transformações.

Landresse (1825) fez uma indicação de classes inferior e superior, serviçais, gente do povo, "bonze" e religiosos, anciãos, rei, mulheres. Como Landresse é baseado em Rodrigues, ele faz uso dos termos "bonze", denominação utilizada pelo padre português para se referir aos monges budistas, e "rei", termo que equivaleria à monarquia europeia, como era utilizado por Rodrigues no século XVII.

Brown (1863) apresenta a polidez por meio de classes sociais (imperador, taikun, nobres, oficiais do governo) e da relação entre falante e seu interlocutor (inferior/ superior/igual). Taikun equivalia ao xogum do clã Tokugawa, que foi abolido com a Restauração Meiji.

Hoffmann (1868) quase não se refere a classes sociais, exceto por meio do termo "prince", que faz referência à pessoa da monarquia. Acredita-se que Hoffmann não distinguia a figura do imperador da dos monarcas europeus. Satow (1872 e 1904[1875]) apenas divide as pessoas em superior, inferior e igual, sem usar as classes sociais preexistentes a 1868. No entanto, se os pronomes apresentados forem analisados, nota-se que Satow apresenta formas utilizadas tanto no Período Edo quanto as formas novas que surgem no Período Meiji. Por isso, pode-se concluir que suas obras representam a fase de transição entre o momento anterior e posterior à Restauração Meiji, isto é, uma transição para a modernização da língua.

Aston (1888[1869] e 1904[1872]) faz uma descrição do uso da polidez de acordo com a posição social do falante (coolies, students, soldiers, lower classes, women) e sua relação com o interlocutor (to inferior, to superior), também sem fazer referência às antigas classes sociais anteriores à Restauração Meiji.

Por fim, Chamberlain (1888) não utiliza essas antigas classes sociais na sua gramática, mas vale-se da divisão em hierarquias de superioridade e inferioridade.

Desse modo, com relação às antigas classes sociais, pode-se perceber que há uma descontinuidade a partir de Satow, que representa uma transição. Assim como Satow, Aston e Chamberlain já não se valem dessas antigas classes sociais, demonstrando estarem descrevendo a língua falada durante o Período Meiji.

\section{Considerações finais}

De acordo com a análise da capa técnica, pode-se concluir que, com relação ao uso da classe de palavras "pronomes", há uma continuidade dessa tradição entre as obras analisadas do século XIX.

De um modo geral, Landresse (1825), por ser uma tradução, representa uma continuidade em relação às obras de Rodrigues (1604), mas está em descontinuidade em relação às obras posteriores, pois descreve a mesma língua falada nos séculos XVI e XVII, que deixou de ser falada nos séculos seguintes. Isso significa que a obra do tradutor francês, apesar de apresentar diferenças com relação ao padre português, não descreve a língua falada pela sociedade japonesa no século XIX. 
A gramática de Hoffmann (1868) é uma obra com características bem peculiares, que destoa das demais obras tanto pelos idiomas descritores usados como pelos métodos de apresentação de dados. Os exemplos de pronomes de $1^{\text {a }}$ pessoa também são bem diferentes dos demais apresentados nas gramáticas analisadas, como se pode ver no Anexo $I$.

Observou-se também que as antigas classes sociais (samurai, agricultor, artesão e comerciante) deixaram de ser mencionadas em Satow (1873 e 1904[1875]), Aston (1888[1869] e 1904[1872]) e Chamberlain (1888). Em vez disso, houve uso de diferenças hierárquicas entre as pessoas descritas como "superiores", "inferiores" e "iguais", o que representa um período de transição social ocasionado pela Restauração Meiji.

Chamberlain (1888), abandonando as expressões antigas, representa uma descontinuidade em relação às obras anteriores.

\section{REFERÊNCIAS}

ASTON, W. G. A Grammar of the Japanese Spoken Language. By W. G. Aston. 4. ed. Tokio: The Hakubunsha; London: Trübner \& Co., Ludgate Hill, 1888 [1869]. Disponível em: $<$ https://archive.org/details/Grammarfjapanes 00astorich>. Acesso em: 11 out. 2015.

. A Grammar of the Japanese Written Language. 3. ed. London: Luzac \& Co., Yokohama: Lane, Crawford \& Co., 1904[1872]. Disponível em: $<$ https://archive.org/details/grammarofjapanes00asto $>$. Acesso em: 15 out. 2017.

BROWN, S. R. Colloquial Japanese, or conversational sentences and dialogues in English and Japanese, together with an English-Japanese index to serve as vocabulary, and an introduction on the grammatical structure of the language. Shangai: Presbyterian Mission Press, 1863.

CHAMBERLAIN. B. H. A Handbook of Colloquial Japanese. London: Trubner \& Co.; Tôkyo: The Hakubunsha; Yokohama, Shanghai, Hong Kong, Singapore: Kelly \& Walshm Limited, 1888.

. A Handbook of Colloquial Japanese. 2. ed. London: Trubner \& Co.; Tôkyô: The Hakubunsha, Ginza. Yokohama, Shanghai, Hong Kong, Singapore: Kelly \& Walsh, Limited, 1889[1888].

COELHO, O.; HACKEROTT, M. M. S. Historiografia Linguística. In: GONÇALVES, A. V.; GÓIS, M. L. de S. Ciências da linguagem: o fazer científico? v. 1. São Paulo: Mercado das Letras, 2012. p. 381-407.

DOI, T. Nihongo no Rekishi (História da língua japonesa). 16. ed. rev. Tóquio: Shibundô, 1979[1957].

FRELLESVIG, B. A History of the Japanese Language. First paperback edition with corrections. Cambridge: Cambridge University Press, 2011[2010].

HOFFMANN, J. J. A Japanese Grammar. Leiden: A. W. Sythoff, 1868.

KIKUCHI, W. Taigû Hyôgen (Expressões de tratamento). In: MORALES, L. M. (Org.). Tópicos de Gramática da Língua Japonesa. Centro de Estudos Japoneses da Universidade de São Paulo. São Paulo: Fundação Japão, 2011. p. 197-217. 
KOERNER, E. F. K. Models in Linguistic Historiography. In: Practicing Linguistic Historiography. Selected Essays. Amsterdam/Philadelphia: John Benjamins Publishing Company, 1989.

RODRIGUES, J. Arte da lingoa de Japam. Edição fac-similar, de 1977, editada pela Benseisha em Tóquio. Nagasaki: Companhia de Jesus, 1604-8.

Éléments de la Grammaire Japonaise, Par le P. Rodriguez. Traduits du Portugais sur le Manuscrit de la Bibliotheque du Roi, et soigneusement collationnés avec la Grammaire publiée par le même auteur à Nagasaki en 1604. Tradução de M. C. Landresse. Paris: Librairie Orientale de Dondey-Dupré Pere et Fils, 1825. Disponível em: $<$ http://www.jlect.com/resources.php $>$. Acesso em: 20 out. 2015.

SATÔ, K. Kokugoshi (História da língua japonesa). v. II. 2. ed. Tóquio: Ofûsha, 1992[1973].

SATOW. E. M. Kuaiwa Hen, twenty-five exercises in Yedo colloquial for the use of students. Yokohama: Lane, Crawford \& Co., 1873. 1904[1875].

. An English-Japanese Dictionary of Spoken Language. 3. ed. Tóquio: Shûeisha,

SHOGAKUKAN. Nihon Kokugo Daijiten (Grande Dicionário da Língua Japonesa). 2. ed. 13 vols. Shogakukan, 2000-2002. Versão digital disponível em Japan Knowledge: $<\underline{\text { http://www.japanknowledge.com> }}$. Acesso em: 20 out. 2015.

SHUNSAKU, N. FUKUZAWA YUKICHI (1835-1901). Prospects: the quarterly review of comparative education (UNESCO: International Bureau of Education), v. XXIII, n. 3/4, p. 493-506, 1993.

SWIGGERS, P. Modelos, métodos y problemas en la historiografia de la linguística. Nuevas aportaciones a la historiografia linguística. Actas del IV Congreso Internacional de la SEHL. La Laguna (Tenerife), 22-25 octubre de 2003, ed. Corrales Zumbado, C.; Dota Luis, J. et al. Madrid: Arco Libros, 2005 [2004]. p. 113-145.

Recebido em: 29/09/2017

Aprovado em: 14/03/2018 
ANEXO I - Quadro: Tratamento dado aos pronomes pessoais de $1^{\text {a }}$ pessoa

\begin{tabular}{|c|c|c|}
\hline \multicolumn{3}{|c|}{ PRONOMES PESSOAIS DE $1^{\text {a }}$ PESSOA } \\
\hline Obra & $\begin{array}{c}\text { Tradução e terminologia usada no tratamento da } \\
\text { polidez }\end{array}$ & $\begin{array}{l}\text { Observação quanto ao } \\
\text { tratamento da polidez }\end{array}$ \\
\hline $\begin{array}{c}\text { Landresse } \\
(1825)\end{array}$ & $\begin{array}{l}\text { §48. Ainsi que nous l'avons dit, les pronoms primitifs } \\
\text { se déclinent comme les substantifs, en ajoutant les } \\
\text { mêmes particules, pour les cas et pour les nombres. } \\
\text { Exemple: } \\
\text { Pronom de la première personne, singulier et pluriel. } \\
\text { Nom. Ware, warewa, je ou moi; warera, nous. } \\
\text { Gen.Wareno ou warega, de moi, ou du mien; warerano } \\
\text { ou wareraga, de nous, ou des nôtres. } \\
\text { Datif. Wareni, wareye, à moi, pour moi; warerani, } \\
\text { wareraye, à nous, pour nous. } \\
\text { Accus. Warewo, warewoba, moi; warerawo, } \\
\text { warerawoba, nous. } \\
\text { Ablat. Wareyori, de moi, par moi; warerayori, de nous, } \\
\text { par nous. } \\
\text { Pronoms de la première personne. } \\
\text { Ware, warera, watakoushi, soregasi; je ou moi, } \\
\text { formules de politesse dont on se sert pour parler aux } \\
\text { autres avec respect, et avec humilité de soi-même. Les } \\
\text { mots ware, warera s'emploient également dans la } \\
\text { langue parlée et dans la langue écrite. Les deux autres, } \\
\text { watakousi et soregasi, ne s'emploient que dans le } \\
\text { langage. } \\
\text { Mi, miga, midomo, midomoraga, kotsi, kotsiga, je ou } \\
\text { moi, pour les inférieurs à l'égard de leurs supérieurs. } \\
\text { Gousò, je ou moi, pour les bonzes ou les religieux. (Ego } \\
\text { indignus.) } \\
\text { Gourò, je ou moi, pour les vieillards. } \\
\text { Tsinga, je ou moi, pronom dont le roi seul peut se } \\
\text { servir. } \\
\text { Miszoukara, warawa, wagami, je ou moi, pour les } \\
\text { femmes seulement. } \\
\text { Wara, worara, je ou moi, pronoms dont se servent les } \\
\text { gens du peuple en parlant entr'eux. }\end{array}$ & $\begin{array}{l}\text { Comparação com a } \\
\text { língua } \\
\text { explicação em latim e } \\
\text { apresentação } \\
\text { pronomes por meio de } \\
\text { casos da língua latina } \\
\text { para indicar a flexão. Uso } \\
\text { dos termos "primeira } \\
\text { pessoa", "singular" e } \\
\text { plural". } \\
\text { Indicação de "classes } \\
\text { inferior" e "superior", } \\
\text { serviçais, gente do povo, } \\
\text { "bonze" e religiosos, } \\
\text { anciãos, rei, mulheres. } \\
\text { Uso dos termos } \\
\text { "respect", "humilité". }\end{array}$ \\
\hline Brown (1863) & $\begin{array}{l}\text { The emperor, for the pronoun of the first person, uses } \\
\text { (朕) Shin, which may signify, subtle, recondite. The } \\
\text { Taikun, or any nobleman of high rank, would use } Y o^{20} \\
\text { (余 or 予) when addressing inferiors, and Watak'shi if } \\
\text { speaking to a superior. To a friend they would say } \\
\text { Sessh'a (拙者) meaning I. Officers of government } \\
\text { would use Sessh'a and watak'shi, in like }\end{array}$ & $\begin{array}{l}\text { Comparação com a } \\
\text { língua inglesa e } \\
\text { apresentação } \\
\text { pronomes utilizando os } \\
\text { termos "primeira } \\
\text { pessoa". Apresentação da } \\
\text { polidez por meio da } \\
\text { classe social e da relação }\end{array}$ \\
\hline
\end{tabular}

${ }^{20}$ A expressão “yo” era utilizada desde o século X para indicar a primeira pessoa referida por um homem. No Período Meiji (1868-1912), essa forma linguística continuou a ser utilizada e significava "eu" com grande grau de respeito. (Dicionário Nihon Kokugo Daijiten, doravante abreviado NKD) 


\begin{tabular}{|c|c|c|}
\hline & $\begin{array}{l}\text { circumstances. Washi is use by persons of inferior } \\
\text { station, when speaking to those under them. The } \\
\text { meaning of Watak'shi }{ }^{21} \text {, is still uncertain. It is the word } \\
\text { most generally used for the first person. Washi }{ }^{22} \text { and } \\
\text { Ore }{ }^{23} \text { are used by the common people for I. } \\
{[\ldots]} \\
\text { The personal pronouns most commonly heard, are } \\
\text { Watak'shi, Washi, and Ore, for the first person, Anata, } \\
\text { Sonohoö, Omae and Temae, for the second, and Ano o } \\
\text { kata, Ano-kata, Ano h'to or Are wa, for the third. Sama } \\
\text { is often added to those for the second person, and in the } \\
\text { vulgar dialect is contracted into san as Omae san, Anata } \\
\text { san. }\end{array}$ & 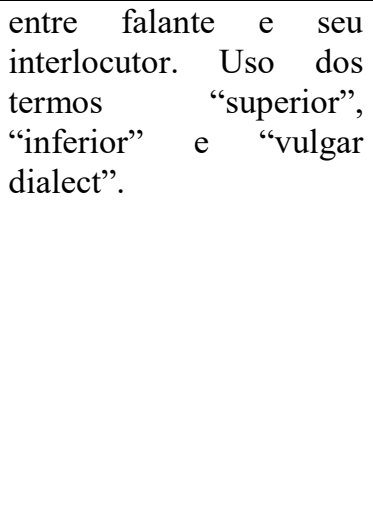 \\
\hline $\begin{array}{c}\text { Hoffmann } \\
(1867-8)\end{array}$ & $\begin{array}{l}\text { 1. For I. } \\
\text { 1) The humblest expression. Is Te-mae, plural Te-mae- } \\
\text { tatsi, in the popular tongue of Yedo which frequently } \\
\text { changes a to e, Temée, =at hand, i.e. that which is at } \\
\text { hand or present to the person opposite. } \\
\text { 2) Watakusi, plural Watakusi-domo, a modest, and, in } \\
\text { confidential intercourse, most usual expression. Every } \\
\text { respectable man speaks of himself thus; and the man of } \\
\text { the people at Yêdo says for it Wasi (uaschi). } \\
\text { 3) Oré, plural Oréra, in the Yêdo-dialect Oira, after the } \\
\text { mention on page } 86 \text { supported by a quotation from the } \\
\text { Dictionary of the old-Japanese language, a self- } \\
\text { humiliating expression., is now considered as one of } \\
\text { pride of Yêdo. } \\
\text { 4) Wáre, plural Warera, the "I" and "We" in the month } \\
\text { of a prince, when he speaks to his people. }\end{array}$ & $\begin{array}{l}\text { Comparação com a } \\
\text { língua holandesa, alemã e } \\
\text { inglesa. Descrição da } \\
\text { língua falada na região de } \\
\text { Yedo. Uso dos termos } \\
\text { "humble", "respectable", } \\
\text { "modest", "confidential } \\
\text { intercourse", "self- } \\
\text { humiliating". Polidez se } \\
\text { expressa de acordo com a } \\
\text { "respeitabilidade" do } \\
\text { falante e a sua relação } \\
\text { com o interlocutor. }\end{array}$ \\
\hline Satow (1873) & $\begin{array}{l}\text { Pronouns of the first person (gradually increasing in } \\
\text { politeness) are: } \\
\text { To inferiors: - oré, pl. oré ra, oira (chiefly among the } \\
\text { lower classes); jibun, pl. jibun domo; washi, pl. washi } \\
\text { domo; konohô, pl. konohô domo; kochi, pl. kochira } \\
\text { (also used as a singular). } \\
\text { To equals (gradually decreasing in politeness), sessha } \\
\text { (somewhat stiff), pl. sessha domo; watakushi, pl. } \\
\text { watakushi domo; boku (much used by students); } \\
\text { watashi, pl. watashi ra (also much used by women); } \\
\text { washi, pl. washi domo. In the intercourse of equals } \\
\text { watakushi, though strictly due only to superiors, is most } \\
\text { employed for I, agreeably to the Japanese custon of }\end{array}$ & $\begin{array}{l}\text { Comparação com a } \\
\text { língua inglesa. Uso do } \\
\text { termo "pronome de } 1^{\text {a }} \\
\text { pessoa" para indicar a } \\
\text { classe morfológica. } \\
\text { Apresentação da polidez } \\
\text { de acordo com a relação } \\
\text { do falante e o } \\
\text { interlocutor: to } \\
\text { inferiors" (entre pessoas } \\
\text { de classes baixas), "to } \\
\text { equals" (entre estudantes, } \\
\text { mulheres) e "to } \\
\text { superiors". }\end{array}$ \\
\hline
\end{tabular}

\footnotetext{
${ }^{21}$ A palavra "watakushi" era usada tanto por homens quanto por mulheres para expressar respeito ao se referirem a si mesmos muito antes do Período Meiji e continua tendo o mesmo sentido até os dias de hoje (NKD).

${ }^{22}$ A expressão “washi”, segundo o dicionário NKD, até meados do Período Edo (1603-1868) era usada por mulheres apenas, mas, a partir do Período Meiji e até os dias atuais, é usada por homens ao se dirigirem a pessoas de nível inferior, manifestando respeito.

${ }^{23}$ A expressão “ore”, a partir do final do Período Edo (1603-1868), passou a ser usada apenas por homens para se referirem a si mesmos na comunicação com pessoas superiores, inferiores e de mesmo nível social, sem expressar vulgaridade (NKD).
} 


\begin{tabular}{|c|c|c|}
\hline & $\begin{array}{l}\text { treating an equal as if he were by one grade a superior. } \\
\text { Anata is used for 'you' in the same way. } \\
\text { To superiors, (gradually increasing in politeness): - } \\
\text { watakushi, pl. watakushi ra or domo; watakushi domo } \\
\text { and watakushi tachi are also used as singulars; temaë, } \\
\text { pl. temaë domo; and temaë no hô dé is often used as a } \\
\text { periphrase for the nominative of this last. }\end{array}$ & $\begin{array}{ll}\text { Uso dos } & \text { termos } \\
\text { "politeness", } & \\
\text { "depreciatory", } & \text { "formal } \\
\text { expression.", } & \text { "vulg.", } \\
\text { "familiar". } & \end{array}$ \\
\hline Satow (1875) & $\begin{array}{l}\text { I, pron. Watakushi; (used by M. P.) hon-in (本員); } \\
\text { (depreciatory) sessha (拙者); (formal expression, used } \\
\text { by officials) honkwan (本官); (used by women) } \\
\text { watashi; atai; (vulg.) washi; ore; oira. [Boku (僕), also } \\
\text { depreciatory, is used in familiar intercourse, } \\
\text { particularly among students; yatsugare and soregashi } \\
\text { are occasionally used; temae is used by inferiors when } \\
\text { addressing superiors]. } \\
\text { We, pron. Watakushi-domo; wareware; warera; } \\
\text { (editorial) waga hai; gojin (吾人). }\end{array}$ & \\
\hline Aston (1869) & $\begin{array}{l}\text { 13. Watakushi, 'I' (plural watakûshi domo, 'we'), is } \\
\text { the ordinary word for the pronoun of the first person. } \\
\text { Ore (plural orera) is less respectful, and is the word } \\
\text { mostly used by coolies, etc., to each other. To inferiors } \\
\text { it is a somewhat haughty word. Students and soldiers } \\
\text { say boku for 'I', waga hai for 'we'. } \\
\text { Temaye is a humble word for 'I', much used by the } \\
\text { lower classes of Tokio in addressing their superior. It is } \\
\text { also used as a pronoun of the second person. Some } \\
\text { people use their surname instead of the personal } \\
\text { pronoun of the first person. } \\
\text { Other words for 'I' are watashi (familiar), watai (by } \\
\text { women), washi (very familiar), wattchi (rustic), sessha } \\
\text { (formal), oira (familiar), jibun (properly 'self'). }\end{array}$ & $\begin{array}{l}\text { Comparação com a } \\
\text { língua inglesa e uso da } \\
\text { classe de palavras } \\
\text { "pronome" de 1a pessoa. } \\
\text { Descrição do uso da } \\
\text { polidez de acordo com a } \\
\text { posição social do falante } \\
\text { (coolies, students, } \\
\text { soldiers, lower classes, } \\
\text { women) e sua relação } \\
\text { com o interlocutor (to } \\
\text { inferior, to superior). } \\
\text { Uso dos termos: } \\
\text { "respectful", "haughty", } \\
\text { "humble", "familiar", } \\
\text { "rustic", "formal". }\end{array}$ \\
\hline Aston (1872) & $\begin{array}{l}\text { PERSONAL PRONOUNS OF THE FIRST PERSON. } \\
\text { 1. - Japanese words } \\
\text { A, wa, are, ware, waro,- The most ancient Japanese } \\
\text { word for "I" is } a \text { or wa. Both these forms are of frequent } \\
\text { occurrence in the Manyôshiu, but they have become } \\
\text { obsolete in the later forms of the language. (...) } \\
\text { Waga has also the meaning "one's own". Waga ko, for } \\
\text { instance, may mean "his or her own child". Wa nami is } \\
\text { properly a plural, but it is often used as a singular. } \\
\text { Are, another obsolete word for "I", is } a \text { with the syllable } \\
\text { re, which is also found in sore, kare, tare, \&c. } \\
\text { Ware (plural* warera, wareware, or waredomo) is } \\
\text { formed by adding the re just mentioned, or waredomo) } \\
\text { is formed by addind the re just mentioned to wa, "I". It } \\
\text { is the most general word for the pronoun of the first } \\
\text { person, and is found in the most ancient as well as in } \\
\text { the latest forms of the language. It is occasialnally } \\
\text { heard in the spoken language in the plural form warera, }\end{array}$ & $\begin{array}{l}\text { A obra de } 1872 \text {, por ser } \\
\text { uma descrição da língua } \\
\text { escrita, distingue-se } \\
\text { completamente da obra } \\
\text { que descreve a língua } \\
\text { oral. Além de trazer } \\
\text { exemplos de termos } \\
\text { usados em obras literárias } \\
\text { desde o século VIII, traz } \\
\text { classes sociais existentes } \\
\text { no passado japonês bem } \\
\text { anterior ao ano de } \\
\text { publicação. } \\
\text { Desse modo, não serve } \\
\text { como comparação para o } \\
\text { estudo dos pronomes } \\
\text { pessoais. }\end{array}$ \\
\hline
\end{tabular}


which is used towards inferiors as a pronoun of the second person plural equivalente to orera. In the modern epistolar style, warera is used for "I" in addressing inferior. Instead of ware no and warega, waga is used.

Waro is an obsolete word for "I". It seems to be only a variation of ware.

Watakushi.- As a pronoun, watakushi belongs to the spoken language, and to modern epistolar correspondence, where it is the commonest word for "I". Watakushi gi (sic) (thing) and watakushi kata (side) are also used. In the literary language watakushi means "selfishness" "that which is private or personal". Its derivation is doubtful.

Maro, - Maro is used chiefly, although not invariably, to inferiors, or to persons with whom one is on terms of close intimacy. It has much the same force as the ore or washi of the spoken language. Towards persons with whom one is not on familiar terms, maro is a haughty word for "I". The author of the Makura Zôshi remarks that the Court nobles, in addressing the Mikado, should not say maro for "I", but should use their own names instead.

In the Tosa Nikki a child says, - "Maro kono uta no kaheshi sen", "I will composse a reply to this verse of poetry". In one of Motowori's works he says: "Aru hito tohite ihaku", "Uta to ha ikanaru mono wo ifu zo ya?" "Maro kotahete ihaku". "A certain person asked of me, 'How do you define poetry?' and I answered...."

The Mikado and persons of high rank are in books made to use maro towards inferiors, as Maro no yo ni, "in my reign". It is little used in the modern written language. Waraha (literally, "the child"), corresponding to the Chinese 妾 (shô), is only used by women. Ex.: Waraha wo moi te yuki tamahe, "take me with you, too".

Onore (plural onorera) is properly of no person. It means "self", "oneself", and may be used indifferently for "myself", "yourself", "herself", and "himself". In practice, however, it is so little different from "I" that a place has been given it among the pronouns of the first person.

In the spoken language, onore is usually of the second person, and is a contemptuous word oftenest heard along with abusive language.

Mi, midomo, midzukara. - Mi literally means "body", "person", and hence "self", but like onore it is often used as a pronouns of the first person. Waga mi has the same meaning as mi. The derived form midzukara is, however, commoner. It is formed by adding to $m i$ the genitive particle $t s u$, and the kara which also appears in nagara, mono kara, \&c. Midzukara means "by or of oneself", "by or of myself".

Midomo is used in the spoken dialect by persons of the samurai class for the singular and plural indiscriminately.

Name of the speaker used instead of the pronoun if the first person.- Both in speaking and in writing, the name 


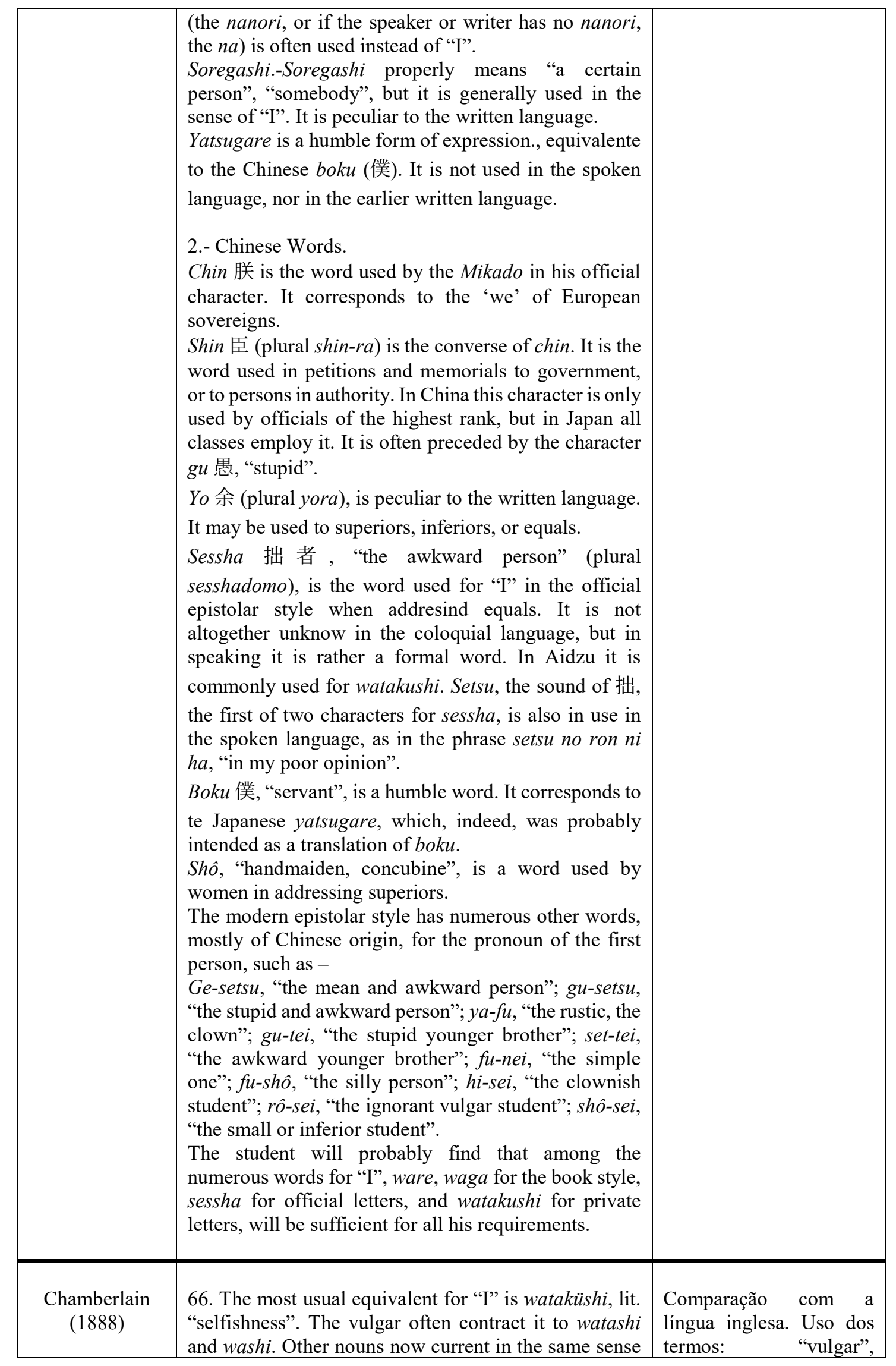




\begin{tabular}{|l|l|l|}
\hline are $b o k u^{24}$, "servant", which is much affected by young & "familiar", "vulgar \\
men in familiarly addressing each other; sessha, "the & corruption". \\
awkward person"; shôsei, "junior". Ore is a very vulgar & Não há divisão nas \\
corruption of ware, which is the commonest word for & antigas classes sociais, \\
& "I" in the Written Language. Orâ, which may often be \\
heard from the mouths of coolies, is for ore wa. & $\begin{array}{l}\text { mas entre expressões de } \\
\text { uso comum, familiar, } \\
\text { vulgar, usados por } \\
\text { serviçais, etc. }\end{array}$ \\
\hline
\end{tabular}

\footnotetext{
24 "Boku", enquanto pronome de tratamento de $1^{\text {a }}$ pessoa do singular, possui registros em textos escritos japoneses anteriores ao Período Meiji (NKD). No Período Edo, há registros nos textos escritos em estilo kanbun (escrita em chinês) de que "boku" era usado por jovens para se referir a si mesmos no diálogo com pessoas de nível inferior ou igual (NKD). A partir do Período Meiji, passou a ser usado, independentemente da idade, por crianças e jovens do sexo masculino para se referir a si mesmos (NKD). Chamberlain, ao apresentar a expressão "boku" como a usada por serviçais revela uma evidência: os serviçais poderiam ser meninos jovens. Essa questão será alvo de nossas investigações futuras.
} 